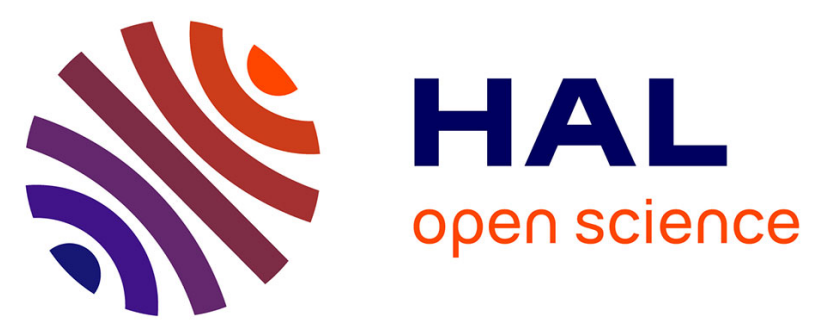

\title{
Insights into Frequency Diversity from Measurements on an Indoor Low Power Wireless Network Testbed
}

\author{
Pedro Henrique Gomes, Ying Chen, Thomas Watteyne, Bhaskar
}

Krishnamachari

\section{- To cite this version:}

Pedro Henrique Gomes, Ying Chen, Thomas Watteyne, Bhaskar Krishnamachari. Insights into Frequency Diversity from Measurements on an Indoor Low Power Wireless Network Testbed. IEEE Global Telecommunications Conference (GLOBECOM), Workshop on Low-Layer Implementation and Protocol Design for IoT Applications (IoT-LINK), Dec 2016, Washington, DC, United States. hal01355060

\author{
HAL Id: hal-01355060 \\ https://hal.inria.fr/hal-01355060
}

Submitted on 20 Dec 2016

HAL is a multi-disciplinary open access archive for the deposit and dissemination of scientific research documents, whether they are published or not. The documents may come from teaching and research institutions in France or abroad, or from public or private research centers.
L'archive ouverte pluridisciplinaire HAL, est destinée au dépôt et à la diffusion de documents scientifiques de niveau recherche, publiés ou non, émanant des établissements d'enseignement et de recherche français ou étrangers, des laboratoires publics ou privés. 


\title{
Insights into Frequency Diversity from Measurements on an Indoor Low Power Wireless Network Testbed
}

\author{
Pedro Henrique Gomes*, Ying Chen ${ }^{\dagger}$, Thomas Watteyne ${ }^{\ddagger}$, Bhaskar Krishnamachari* \\ *University of Southern California, Los Angeles - USA, email: \{pdasilva,bkrishna\}@usc.edu \\ †VMware, Palo Alto - USA, email: yingchen@ vmware.com \\ ‡Inria, EVA team, Paris - France, email: thomas.watteyne@inria.fr
}

\begin{abstract}
Recent wireless medium access control techniques, such as the Timeslotted Synchronized Channel Hopping (TSCH) and Deterministic \& Synchronous Multi-channel Extension (DSME) modes in the IEEE802.15.4-2015 standard, use frequency diversity to cope with external interference and multipath fading. The result is wire-like reliability in a network built from unreliable wireless links. Yet, the impact of using multiple frequencies on the medium access control layer is still not perfectly understood, and virtually all channel hopping solutions use "blind" channel hopping, i.e., hopping over all frequencies equivalently. The goal of this work is to improve our understanding of the behavior of the wireless medium when using multiple frequencies, which will enable the design of more efficient protocols in the future. We collect a large dense connectivity dataset over the USC Tutornet Internet of Things Testbed, with dozens of low-power wireless nodes deployed in an office building. This publicly-available dataset offers complete traces of link quality across frequency, time and space. We analyze the data and extract meaningful and practical insights on the wireless medium when using multiple frequencies.
\end{abstract}

Index Terms-Wireless Sensor Networks, Diversity, Testbed.

\section{INTRODUCTION}

Low-power wireless networks, including wireless sensor networks (WSNs), are composed of devices with constrained processing capability, memory, energy and communication. Many of these devices are equipped with a low-power wireless transceiver compliant to the IEEE802.15.4 [1] standard, and operating on the $2.4 \mathrm{GHz}$ frequency band. The presence of other technologies on that band, the low-power nature of the sensors and other constraints cause these networks to be composed of unreliable and time varying wireless links. As a result, IEEE802.15.4-based networks that simply rely on CSMA/CA are not scalable and cannot be used in applications where high availability and quality of service are prerequisites [2]. Yet, low-power wireless networks are increasingly being envisioned to wirelessly interconnect the Internet of Things (IoT), which is expected to connect billions of devices and cover a large variety of applications, from

This work was supported in part by NSF grants CCF-1423624 and AST1248017, by Inria through the DIVERSITY associate team, and the European Commission through the F-Interop and ARMOUR H2020 projects. It was done while Ying Chen was at the University of Southern California.

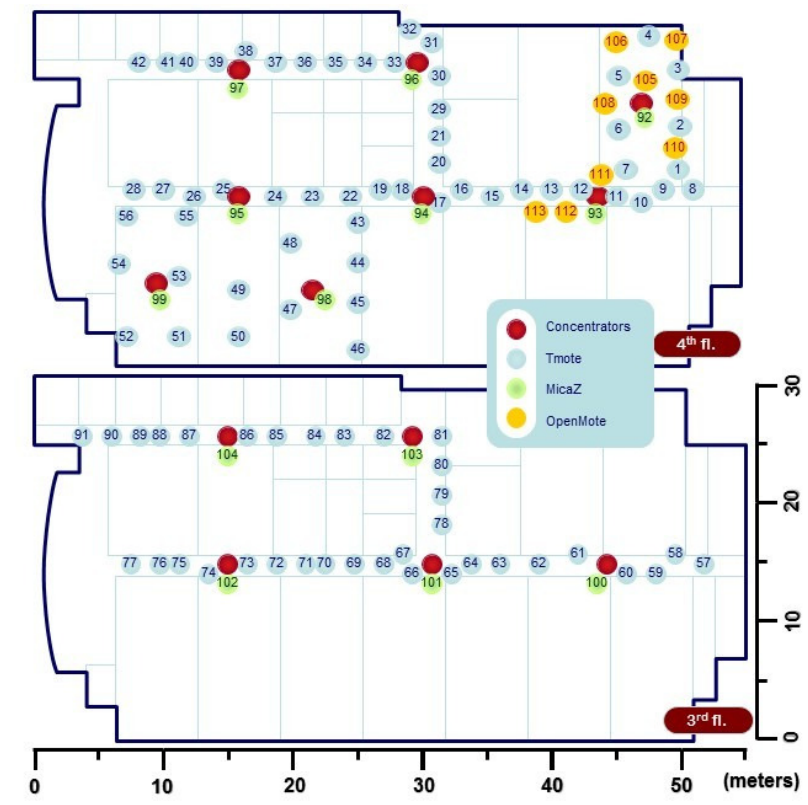

Fig. 1. The Tutornet testbed is deployed over 2 stories in an office building on the USC campus.

home automation to smart factories. It is therefore important to develop robust low-power wireless protocols which can cope with the unreliable nature of the wireless links.

Exploiting diversity is key to building a reliable lowpower wireless network. Multi-channel protocols that exploit frequency diversity has three main benefits: (i) it makes the network more robust against external interference and multi-path fading, thereby increasing its reliability [3], (ii) it reduces congestion and medium contention [4], [5], and (iii) it increases the overall network throughput by allowing concurrent transmissions on orthogonal channels [6], [7].

A key point in frequency-agile techniques is the policy for choosing which of the frequencies ${ }^{1}$ to use at a given point in time. The $2.4 \mathrm{GHz}$ band is shared with other networks

\footnotetext{
1 Throughout this paper, the terms "channel" and "frequency" are used interchangeably.
} 
such as WiFi, Bluetooth and RFID, as well as microwaves and industrial/medical equipment. It is important to better understand how different frequencies are correlated (or not), to design better multi-channel protocols. Interference and multipath fading effects highly depend on the environment and are hard to predict in general.

This paper introduces a large, comprehensive and dense dataset collected experimentally in a real indoor "Smart Building" IoT testbed consisting of 113 nodes deployed across two floors. This dataset is dense in time, space and frequency. We are making it freely available for use by researchers. We extract from the dataset important insights about the performance of multi-channel low-power wireless solutions. Insights include how different the performance is on different channels, how many channels to use, and what is the duration of link fadings.

The remainder of this paper is organized as follows. Section II surveys significant related work on multi-channel protocols. Section III presents Tutornet, an indoor low-power wireless IoT testbed. Section IV details the dense connectivity dataset used in our analysis. Section V analyzes the obtained results. Finally, Section VI concludes the paper.

\section{RELATED WORK}

Early work on Wireless Sensor Networks (WSNs) focused on collection applications and were based on single-channel schemes that run over CSMA/CA or encounter-based medium access. Some studies empirically analyze the performance of wireless links, demonstrating the main reasons for wireless unreliability [8], asymmetry [9] and other factors that affect the packets delivery ratio of low-power wireless networks [10]. These works, however, focus on the study of single-channel networks and do not analyze link statistics across multiple channels, which is useful for multi-channel protocols.

As a solution for many limitations found in single-channel networks, multi-channel operation has been a common approach used by most recent works. A comprehensive survey on multi-channel protocols can be found in [11]. We present below a summary of this related work, and analyze how a better understanding of dynamics across different frequencies contributes to that related work.

"Multi-frequency Media access control for wireless Sensor Networks" (MMSN [5]) protocol is, to the best of our knowledge, the earliest multi-channel MAC proposal for WSNs with half-duplex transceivers. In MMSN, each node is assigned a single frequency that it uses for receiving data. All nodes are synchronized and use a portion of the timeslots to communicate using a single broadcast frequency, and the rest using multiple unicast frequencies. Even though multiple frequencies are employed, MMSN does not use frequency hopping.

Y-MAC [4] presents a more sophisticated scheme for saving energy. Y-MAC also operates on timeslots, however, instead of assigning a receive frequency for each node, it assigns a receive timeslot and all nodes initially use the same base frequency. When a transmission occurs, the protocol starts a frequency hopping algorithm. This way, whenever a data burst

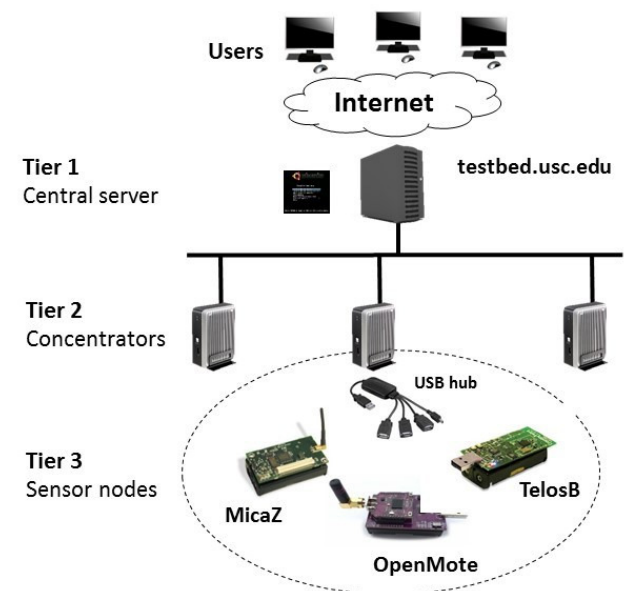

Fig. 2. The architecture of the Tutornet IoT testbed.

occurs, all transmissions are scheduled in multiple frequencies using a pseudo-random sequence.

The "Packets in Pipe" (PIP [6]) protocol is a bulk-transfer that creates a multi-channel time-slotted schedule for optimizing throughput. PIP acts as a transport-layer protocol that establishes a multi-hop path towards the sink. The path and schedule construction is executed at the sink and utilizes multiple frequencies to achieve maximum throughput, with parallel transmissions.

$P^{3}$ [7] enhances several aspects of PIP and proposes a near-optimal bulk-transfer protocol, exploiting both space and frequency diversity. In each timeslot, multiple nodes transmit and receive the same packet simultaneously. This way, in addition to increasing SNR through constructive interference, $\mathrm{P}^{3}$ is able to increase the probability of packet reception, since receivers at different locations suffer from non-correlated interference. In order to prove the efficiency of the proposal, the authors in [7] conduct two studies that analyze channel quality differences and correlation among packet reception. To the best of our knowledge, $\mathrm{P}^{3}$ is one of the only prior work in the literature that conducts such analysis and shows the important role that channel quality statistics play in the development and evaluation of multi-channel protocols.

MultiChannel Collection protocol (MCC) [12] proposes a complete solution for the optimization of WSNs focusing on data collection. It proposes heuristics for the formation of routing trees, slots scheduling, and frequency allocation in multi-hop multi-channel networks. MCC is the earliest protocol to propose a solution for data collection that ensures fairness among all nodes and yet provides high throughput.

Finally, Time Synchronized Mesh Protocol (TSMP [3]) forms the core used in the WirelessHART, ISA100.11a and IEEE802.15.4 TSCH standards. It has been employed in critical WSNs and industrial applications, mainly because of its throughput efficiency and robustness against external interference. TSMP is based on timeslots and uses multiple frequencies with the aid of frequency hopping spread spectrum (FHSS) to exploit frequency diversity.

From all the mentioned multi-channel protocols, it is clear 
TABLE I

THE THREE GENERATIONS OF MOTES DEPLOYED ON TUTORNET.

\begin{tabular}{||cccccccc||}
\hline Mote & Manufacturer & Year & Microcontroller & Flash size & RAM size & Transceiver \\
\hline \hline MicaZ & CrossBow & 2004 & Atmel 8-bit ATmega128L @ 8 MHz & $128 \mathrm{kB}$ & $4 \mathrm{kB}$ & CC2420 \\
\hline TelosB & MoteIV & 2005 & TI 8-bit MSP430F1611 @ 8 MHz & $48 \mathrm{kB}$ & $10 \mathrm{kB}$ & CC2420 \\
\hline OpenMote & OpenMote & 2015 & TI 32-bit CC2538 @ 32 MHz & $512 \mathrm{kB}$ & $32 \mathrm{kB}$ & $2.4 \mathrm{GHz}$ SoC \\
\hline
\end{tabular}

that a better understanding of the link quality over multiple frequencies is beneficial for the development and optimization of these networks. The protocols that use a base frequency for negotiation or broadcast - such as MMSN [5] and Y-MAC [4] - can pick the frequency that yields the best performance. Protocols that assign static frequencies to nodes or for each hop along the path - such as MMSN [5], PIP [6], $\mathrm{P}^{3}$ [7] and MCC [12] - can decide the best frequency to be assigned based on the location of the nodes and the spatial dynamics of link quality. Finally, FHSS-based protocols - such as TSMP [3] and Y-MAC [4] - can benefit from the temporal and spatial link dynamics to select the best frequencies to be used in the hopping sequence. These observations motivate our work.

\section{TUtORnet - A LOW-POWER Wireless IOT TESTBED}

Tutornet is a low-power wireless network testbed deployed at the Ming Hsieh Department of Electrical Engineering at the University of Southern California (USC). It is extensively used for research and teaching at USC. Tutornet is composed of 113 sensor nodes (91 TelosB, 13 MicaZ, 9 OpenMote) covering two adjacent floors in an office building ${ }^{2}$.

Tutornet has a three-tier heterogeneous [13] architecture depicted in Fig. 2. At Tier 1, a central server is responsible for the testbed reservation system and for redirecting communication between the sensor nodes and the remote user. At Tier 2, 13 concentrator nodes are physically connected through USB cables to the sensor nodes, and through Ethernet cables to the central server. At Tier 3, 113 sensor nodes use USB cables as a communication back-haul and as power source.

The central server runs Ubuntu Linux, the reservation system is an Apache-based web server with software written in Perl+MySQL for controlling users privileges. The concentrator nodes are Raspberry-Pi-like computers running Ubuntu Linux. Each concentrator has a USB-hub supporting up to 10 devices. All concentrators are connected to the central server using a high-speed Ethernet network based on Power-Line Communication (PLC) protocol.

Tutornet features three generations of wireless sensor nodes (Table I), all with IEEE802.15.4-compliant radios operating on the $2.4 \mathrm{GHz}$ frequency band.

Fig. 1 shows the layout of the two floors with the arrangement of the sensor and concentrator nodes.

\section{Dense Connectivity Datasets}

We focus on the behavior of IEEE802.15.4-compliant radios operating in the $2.4 \mathrm{GHz}$ frequency band, arguably the most

\footnotetext{
2 Tutornet is managed by the Autonomous Networks Research Group, more information at http://anrg.usc.edu/www/.
}

used technology in low-power wireless. On this band, there are 16 orthogonal frequencies, numbered 11 to 26 , with a bandwidth of $2 \mathrm{MHz}$ and central frequencies separated by $5 \mathrm{MHz}$. Our goal is to quantify how, in this Smart Building deployment, external interference and multi-path fading affect different frequencies, and how the quality of a particular frequency evolves over time and space.

The experiments are performed with the TelosB motes located on the 4th floor, on an area of $55 \times 30$ meters (Fig. 1). On that floor, there are 7 laboratories and 10 offices, all separated by dry-wall. There are $8 \mathrm{WiFi}$ access points spread on the floor, operating across the $2.4 \mathrm{GHz}$ frequency band. Various other sources of interference are present, including Bluetooth devices and micro-wave ovens. An average of 60 people work on this floor daily. External interference and multi-path fading effects are hence very present in this testbed, which is representative of a "Smart Building" deployment.

The firmware used in the experiments is based on TinyOS 2.x and ContikiOS 3.0, and uses simple broadcast communication with no link-layer retransmissions. All packets have a MAC payload size of 100 bytes and are transmitted at $-15 \mathrm{dBm}$ radio transmit power to increase the number of hops in the network, keep connectivity and save energy in real deployments. The experiments are divided in two sets. The first set focuses on a coarse-grained measurement of link quality. We use 55 motes on the 4th floor, each broadcasting 100 packets with $50 \mathrm{~ms}$ inter-packet intervals in a roundrobin fashion, while all other nodes record the total number of packets received, as well as the RSSI and LQI of each. This procedure is executed for all 16 frequencies and repeated 4 times during different days of the week at different times during business hours. The second set focuses on fine-grained analysis. We use 40 nodes, and reduce the inter-packet space to $8 \mathrm{~ms}$. Only the total number of received packets is recorded by all nodes. The full experiment is repeated every $15 \mathrm{~min}$ for 2 days. We obtain a total of 192 snapshots of network statistics on all 16 frequencies in the second set of data ${ }^{3}$.

\section{InSIGHTS INTO MULTI-CHANNEL OPERATION}

The dataset collected contains a wealth of information. In particular, since the data is dense in frequency, it allows us to get insights on how the wireless medium behaves when using multiple frequencies, and make recommendations for designing frequency-agile protocols. We ask ourselves 6 fundamental questions related to multi-frequency operation and use the dataset to answer them.

\footnotetext{
${ }^{3}$ As an online addition to this paper, the complete dataset is available at http://anrg.usc.edu/www/tutornet/.
} 


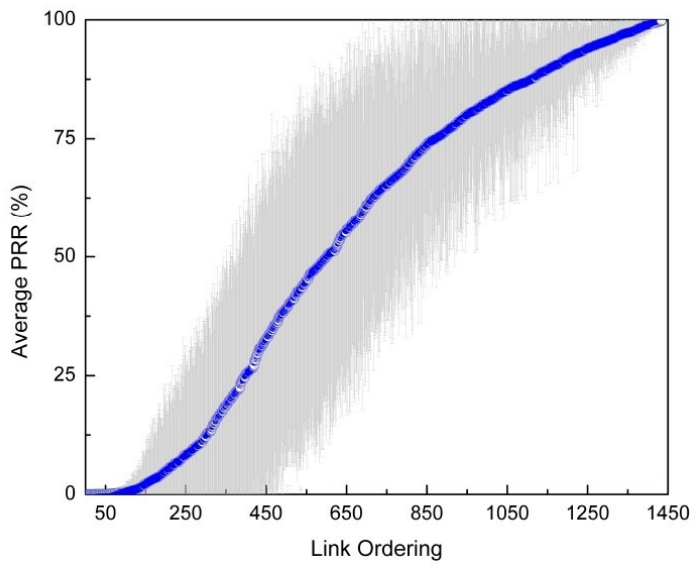

Fig. 3. [Q1] Sorted average PRR over all 16 channels for 1434 links. The error bar represents the standard deviation.

[Q1] How is the distribution of PRR (Packet Reception Ratio) over all 16 channels?

We consider the analysis of unidirectional links $(i, j)$ from node $i$ to node $j$, and define $P R R_{(i, j)}^{c h}$ as the Packet Reception Ratio (PRR) of link $(i, j)$ on channel $c h$. In the 55-node network, with power of $-15 \mathrm{dBm}$, there are 1434 links with $P R R_{(i, j)}^{c h}>0$ in at least one channel. To understand the diversity of link quality in different channels, for each link, we calculate the average PRR on all 16 channels. We sort all links according to their average PRR, and plot the results in Fig. 3 with standard deviation as error bars. Fig. 3 shows that part of the links (the ones with smallest and largest PRR) do not show large variation of quality across different channels. However, links with intermediate average PRR suffer much larger variation over channels and, hence, the choice of the operating channel for these links is especially important.

For reliable transmissions, we should not take into account links with poor quality. We therefore focus our analysis on links with good quality (called good links), which we define as links with $P R R_{(i, j)}^{c h}>\theta$ in at least one channel. We use $\theta=90 \%$. Using this threshold, we find 863 good links and show their average PRR in Fig. 4. We see that, even for links with high PRR in at least one channel, the variation of PRR is very large. In other words, even if a link has a low PRR in some frequency, it is very possible that the PRR is good at another frequency. To put in another way: if node $A$ sends a packet to $B$ at a particular frequency, and $B$ does not receive the packet, node $A$ should retransmit on a different frequency. This is the base idea behind channel hopping.

\section{[Q2] What is the best channel to use?}

For each link, and at any point in time, there is a frequency for which the PRR is highest across frequencies. We define channel $c h^{*}$ as the "best" channel for a link $(i, j)$ if $\operatorname{PRR}_{(i, j)}^{c h^{*}}=\max \left(P R R_{(i, j)}^{c h}, \forall c h \in[11: 26]\right)$. Fig. 5 shows the histogram of $c h^{*}$ for all links and for the good links (see Q1).

One observation is that $c h^{*}$ spreads across all channels, but some channels are more often the best. In the good link case,

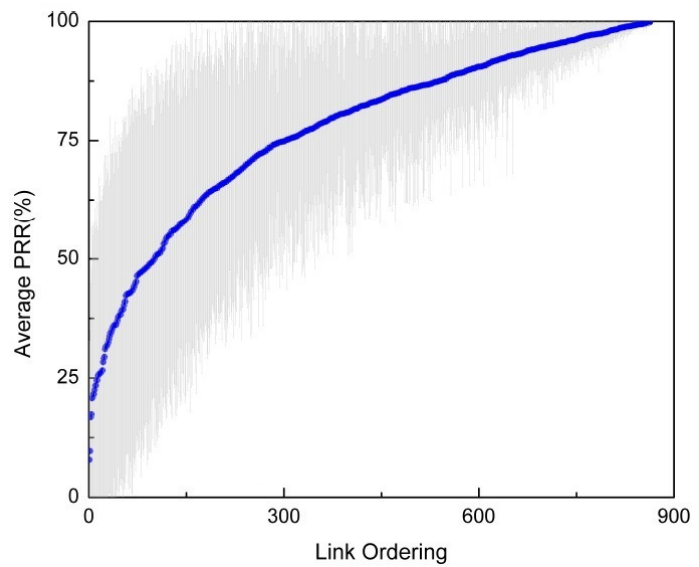

Fig. 4. [Q1] Sorted average PRR in 16 channels for 863 links with $P R R \geq 90 \%$ in at least one channel. The error bar represents the standard deviation.

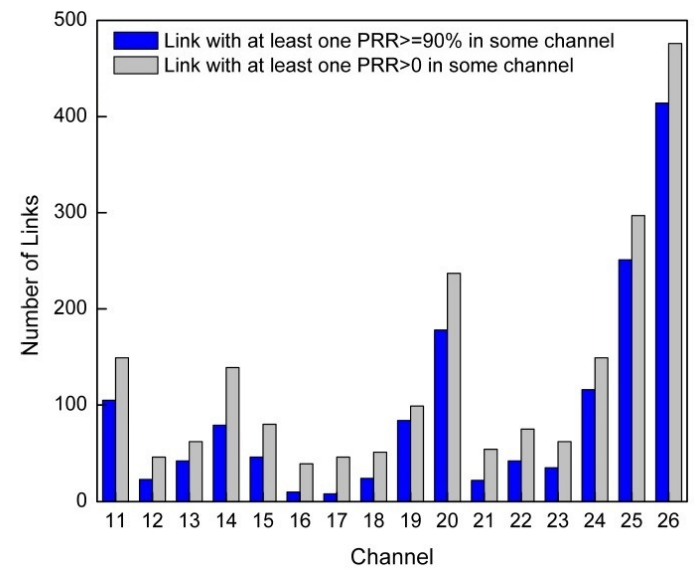

Fig. 5. [Q2] Best channel histogram of links in the 55-node network.

414 out of the 863 links have channel 26 as their best channel, while only 8 links have channel 17 . In general, channels 20,25 and 26 exhibit better average quality, because these channels experience less WiFi interference. Yet, deciding to run an entire network on channel 26 may not be the best solution, since multi-path fading affects all frequencies. Channel hopping, in which every frequency could be used some portion of the time, is the right approach.

[Q3] How many channels should I use when channel hopping is employed?

Most channel hopping solutions use "blind", i.e., hopping across all 16 channels equivalently. The question we ask ourselves is: could we blacklist some channels and hop only on the remaining ones to increase performance? How many channels should remain in the whitelist?

Fig. 6 shows the good channel distribution for good links. We call good channels those channels with $P R R \geq 90 \%$, and good links those links with at least one good channel. From Fig. 6, we can see that in all channels, there are a number of good links; even for channel 15, we have 281 good links. Also, each link might have multiple good channels. If we sum up the numbers of links in all channels, the total is 6643. That is, on 


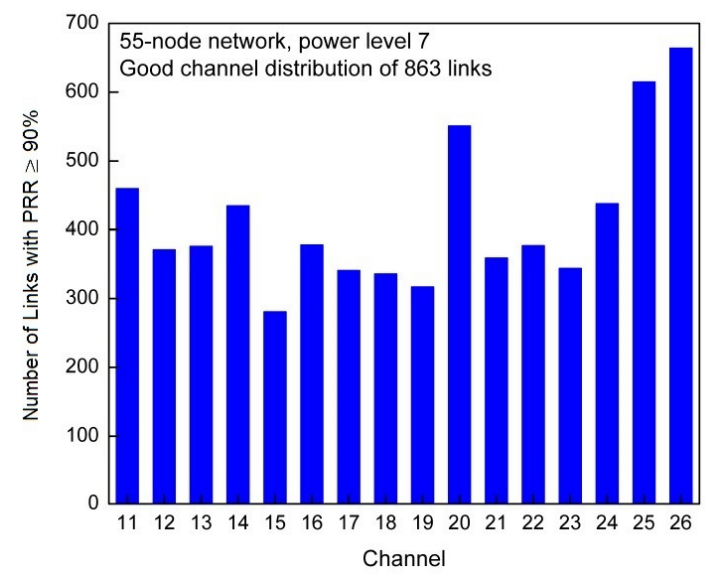

Fig. 6. [Q3] The number of links with $P R R \geq 90 \%$ for each channel.

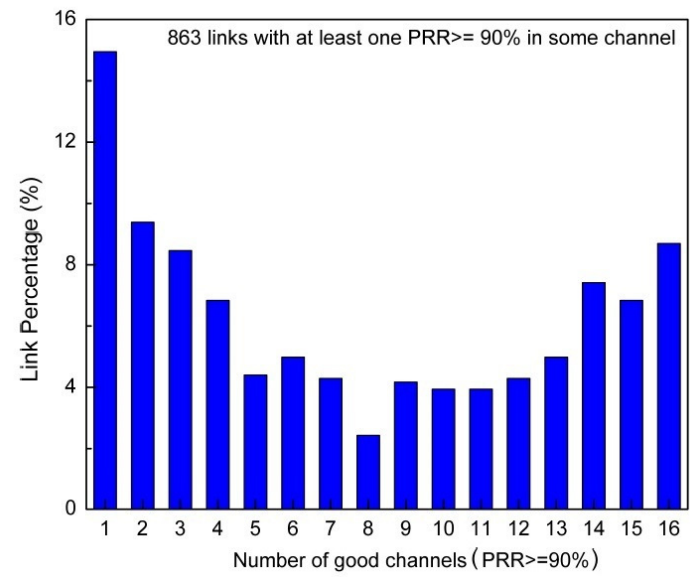

Fig. 7. [Q3] Distribution of the number of channels with $P R R \geq 90 \%$.

average, each link has 6643/863 = 7.7 good channels, which should be the average number of channels to be considered in a multi-channel protocol.

We calculate the number of good channels for each link and show the distribution in Fig. 7. 8.7\% of the links (75 links) have PRR above $90 \%$ in all channels. And only $14.9 \%$ of the links have only 1 channel with PRR above 90\%. The channel allocation for these links is more restricted if we want to guarantee their high delivery ratio.

\section{[Q4] How is the distribution of neighbors with good links?}

We analyze the receiver-based link quality. Especially considering gradient routing, this analysis helps the tree formation algorithm. For a receiver node $i$, we define a node $j$ as its neighbor on channel $c h$ if $P R R_{(i, j)}^{c h}>\theta$. We consider $\theta=90 \%$ and show in Fig. 8 the number of neighbors for each node, and for each frequency.

Fig. 8 illustrates how the channel assignment can drastically change the connectivity and performance of the network. Considering node 17 , if communicating on channel 26 , it has 24 neighbors, but no good neighbors if using channel 15. Knowing the location of the WiFi access points, we remark that sensors close to a WiFi access point have a larger

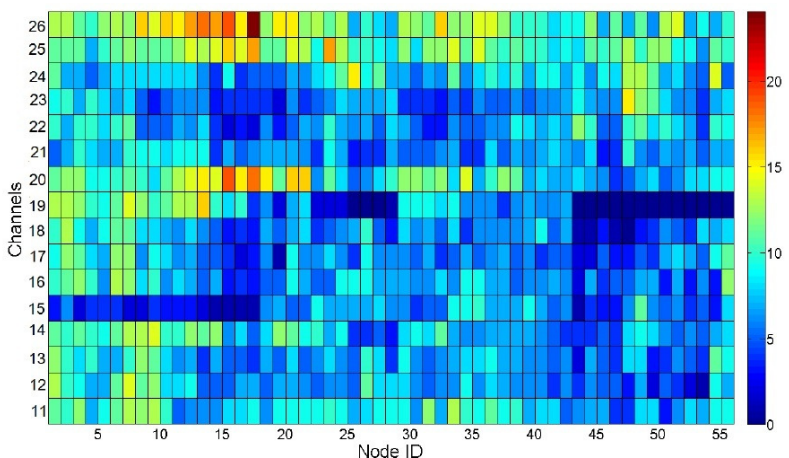

Fig. 8. [Q4] Number of neighbors for each node, for each channel.

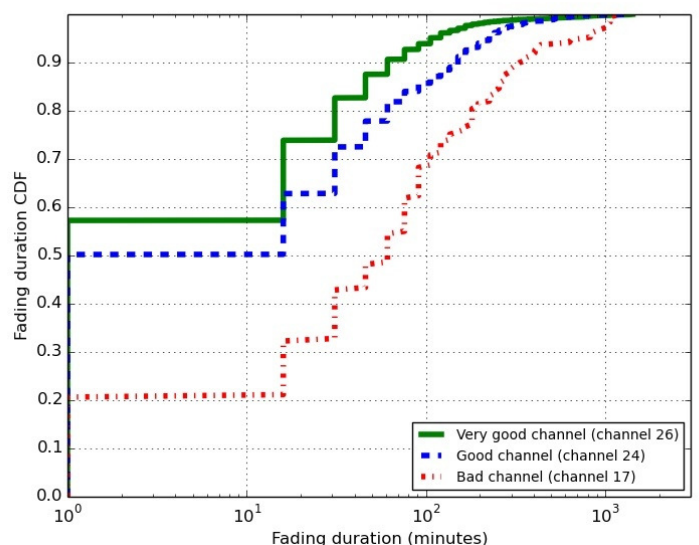

Fig. 9. [Q5] Cumulative distribution function of fading duration.

variation in the number of neighbors over frequency. Hence, nodes closer to interference source suffer larger variation on the number of neighbors.

This highlights the extreme dynamism of the network on a particular channel. Channel hopping stabilizes the topology by averaging the number of neighbors across of frequencies.

\section{[Q5] How long does a link fade last?}

People moving around, doors being opened and closed cause the PRR of links to change over time. We are interest in longterm changes in link PRR (at least $15 \mathrm{~min}$ ). The question we want to answer is: on a given frequency, once the PRR of a link below 90\%, how long does it take for that PRR to be above $90 \%$ ? We call that duration the "fading duration".

Fig. 9 shows the cumulative distribution function (CDF) of the fading duration for 3 different channels. Channel 26 exhibits the shortest fades, channel 17 the longest, channel 24 intermediate. Other channels are not depicted not to overload Fig. 9.

On channel 26 (the channel with the highest link quality), about $60 \%$ of the fades last less than 15 min. Overall considering all channels, $90 \%$ of the fading durations are less than 5 hours. These results corroborate [14], in that link quality shows large variation not only over frequency, but also over time. 


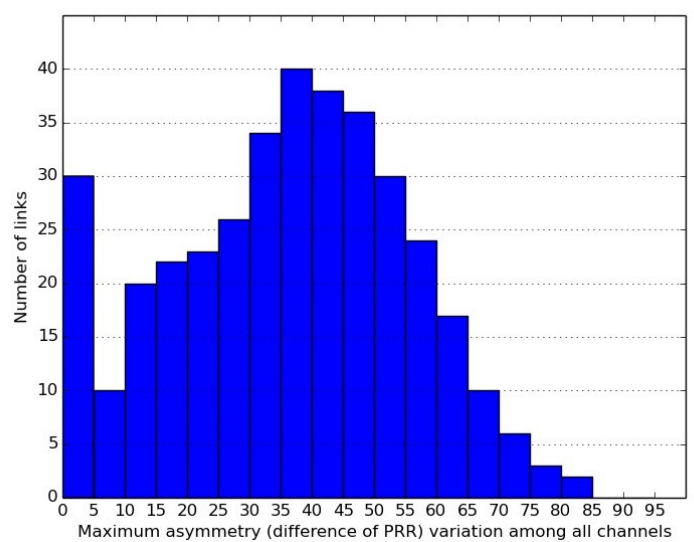

Fig. 10. [Q6] Histogram of maximum asymmetry variation across all 16 channels.

\section{[Q6] Does link asymmetry change across channels?}

Link asymmetry is a well understood phenomenon [9]. It is the difference of PRR between both directions of a given link. To the best of our knowledge, no related work analyzes how frequency affects asymmetry. Asymmetry is caused by differences in transmit power and sensitivity of neighbor nodes. Even if all nodes had the same hardware, differences in manufacturing or aging can cause asymmetry, and difference in electronic components (such as filters with different frequency response) can cause frequency-dependent asymmetry.

We re-define a link $(i, j)$ as a bidirectional connection; its quality is given by the PRR from $i \rightarrow j$ and $i \leftarrow j$ in each channel. We define the asymmetry level $(a l)$ as the absolute difference between the quality of the link on both directions $\left(a l=a b s\left(P R R_{(i, j)}^{c h}-P R R_{(j, i)}^{c h}\right)\right)$. We define the variation of the level of asymmetry as the difference between asymmetry levels on different channels for the same link. For all 301 bidirectional links $\left(P R R_{(i, j)}^{c h}>0\right.$ or $\left.P R R_{(j, i)}^{c h}>0\right)$ in the 40-node network, we calculate the maximum asymmetry level variation. Results are presented in Fig. 10.

Asymmetry does change with frequency. In most links, the variation of asymmetry level across channels is at least $25 \%$. It means that changing the operating channel can easily turn a asymmetric link (with difference of PRR greater than 25\%) into a symmetric link (difference of PRR close to $0 \%$ ), or vice-versa. We conclude that hopping over multiple frequency can also be effective for smoothing the effects of asymmetry on the network protocols.

\section{CONCLUSIONS}

This paper introduces Tutornet, a 113 low-wireless mote testbed deployed across two floors in an office building. We gathered dense connectivity traces measuring the PRR across all 16 frequencies available to the IEEE802.15.4-compliant radios on the $2.4 \mathrm{GHz}$ frequency band. This dense dataset contains a wealth of information, and is made available to the community for conducting further studies.
For this paper, we are particularly interested in better understanding the impact of the wireless channel on medium access protocol running on the devices when using multiple frequencies. We show that, for the same two neighbor nodes communicating, the PRR may exhibit large variation in different frequencies. Part of this is due to external interference (WiFi mainly in the testbed), but even IEEE802.15.4 channels at the same frequency as WiFi channels exhibited a PRR $\geq 90 \%$. This paper hence advocates the use of channel hopping. Instead of picking a single channel and running the complete network on it, we recommend to select multiple channels and use "channel hopping" as a way to cope with external interference and multi-path fading. Blind channel hopping (using all 16 channels) is the simplest approach, but selecting the best 7.7 channels (on average) for each link would yield even better results ${ }^{4}$. We show that, when using multiple channels, the network is less dynamic.

\section{REFERENCES}

[1] "802.15.4-2015 - IEEE Standard for Low-Rate Wireless Personal Area Networks (WPANs)," 2015.

[2] G. Anastasi, M. Conti, and M. Di Francesco, "A Comprehensive Analysis of the MAC Unreliability Problem in IEEE 802.15.4 Wireless Sensor Networks," IEEE Transactions on Industrial Informatics, vol. 7, no. 1, pp. 52-65, 2011.

[3] K. Pister and L. Doherty, "TSMP: Time synchronized mesh protocol," IASTED Distributed Sensor Networks, pp. 391-398, 2008.

[4] Y. Kim, H. Shin, and H. Cha, "Y-MAC: An Energy-Efficient Multichannel MAC Protocol for Dense Wireless Sensor Networks," in Int'l Conf. on Information Processing in Sensor Networks (IPSN), 2008.

[5] G. Zhou, C. Huang, T. Yan, T. He, J. A. Stankovic, and T. F. Abdelzaher, "MMSN: Multi-Frequency Media Access Control for Wireless Sensor Networks," in International Conference on Computer Communications (INFOCOM). IEEE, 2006, pp. 1-13.

[6] B. Raman, K. Chebrolu, S. Bijwe, and V. Gabale, "PIP: A ConnectionOriented, Multi-Hop, Multi-Channel TDMA-based MAC for High Throughput Bulk Transfer," in ACM Conference on Embedded Networked Sensor Systems (SenSys), 2010, p. 15

[7] M. Doddavenkatappa and M. Choon, "P3: A Practical Packet Pipeline using Synchronous Transmissions for Wireless Sensor Networks," in Int'l Conf. on Information Processing in Sensor Networks (IPSN), 2014.

[8] K. Srinivasan, P. Dutta, A. Tavakoli, and P. Levis, "An Empirical Study of Low-power Wireless," ACM Transaction on Sensor Networks, vol. 6 , no. 2, pp. 16:1-16:49, Mar. 2010.

[9] M. Z. Zamalloa and B. Krishnamachari, "An Analysis of Unreliability and Asymmetry in Low-power Wireless Links," ACM Transactions on Sensor Networks, vol. 3, no. 2, Jun. 2007.

[10] J. Zhao and R. Govindan, "Understanding Packet Delivery Performance in Dense Wireless Sensor Networks," in International Conference on Embedded Networked Sensor Systems (SenSys). ACM, 2003, pp. 1-13.

[11] O. D. Incel, "A Survey on Multi-channel Communication in Wireless Sensor Networks," Computer Netw., vol. 55, no. 13, pp. 3081-99, 2011.

[12] Y. Chen, P. H. Gomes, and B. Krishnamachari, "Multichannel Data Collection for Throughput Maximization in Wireless Sensor Networks," in Int.l Conf. Mobile Ad hoc and Sensor Systems (MASS), 2014.

[13] A. Gluhak, S. Krco, M. Nati, D. Pfisterer, N. Mitton, and T. Razafindralambo, "A Survey on Facilities for Experimental Internet of Things Research," IEEE Comm. Mag., vol. 49, no. 11, pp. 58-67, 2011.

[14] T. Watteyne, A. Mehta, and K. Pister, "Reliability Through Frequency Diversity: Why Channel Hopping Makes Sense," in Symposium on Performance Evaluation of Wireless Ad Hoc, Sensor, and Ubiquitous Networks (PE-WASUN). ACM Press, 2009, p. 116.

\footnotetext{
4 This is a theoretical statement only, as a practical solution would require regular probing of all frequencies, which comes at a cost.
} 